\title{
Le système d'information comptable des dirigeants de PME syriennes : complexité et contingences ${ }^{1}$
}

Chapellier P., Mohammed A., Teller R. (2013), «Etude du système d'information comptable des dirigeants de PME syriennes : complexité et contingences », Revue Management et Avenir, $\mathrm{N}^{\circ} 65$, novembre, p.48-72, [AERES C / CNRS cat.4 / CNU C / FNEGE rang 4]

Philippe Chapellier est Maître de Conférences HDR en Sciences de Gestion à l'Institut Universitaire de Technologie de Montpellier et membre du laboratoire «Montpellier Recherche Management» à l'Université Montpellier 2. Ses travaux portent sur les outils de gestion du dirigeant de PME, la relation entre l'expert externe et le dirigeant de PME, et le transfert des connaissances.

Adresse : $\quad$ IUT de Montpellier - Département Informatique

99, avenue d'Occitanie

34296 Montpellier cedex 5

Téléphone : Bureau : 0499585173

Domicile : 0467666256

Courriel : Philippe.Chapellier@univ-montp2.fr

Abdallah Mohammed est Maître de Conférences en sciences de gestion à l'Université d'Alep en Syrie et membre du Centre de Recherche en Ingénierie Financière et Finances Publiques (CRIFP) à l'Université de Nice Sophia Antipolis. Ses travaux portent sur le thème des outils de gestion du dirigeant de PME, leurs spécificités et leurs déterminants.

Adresse : Université d'Alep - Faculté d'Economie

Alep - Syrie

Téléphone : 00963956567713

Courriel : Abdallah_m76@yahoo.com

Robert Teller est Professeur des Universités en Sciences de Gestion et membre du Centre de Recherche en Ingénierie Financière et Finances Publiques (CRIFP) à l'Université de Nice Sophia Antipolis. Ses travaux portent sur la gouvernance des organisations et sur les outils et normes comptables.

Adresse : IAE de Nice

24 Avenue de Diables Bleus

06000 Nice

Téléphone : 0492157088

Courriel : Robert.Teller@unice.fr

\footnotetext{
${ }^{1}$ Ce papier est une version adaptée d'un article intitulé «Les pratiques comptables des dirigeants de PME syriennes dans un contexte de libéralisation de l'économie » présenté au $31^{\text {ème }}$ Congrès de l'Association Francophone de Comptabilité intitulé «Crises et nouvelles problématiques de la valeur » à Nice en mai 2010.
} 


\section{Le système d'information comptable des dirigeants de PME syriennes : complexité et contingences The accounting information system of the managers of syrian SMEs: complexity and contingencies}

Résumé : L'étude, réalisée par administration d'un questionnaire auprès de 92 dirigeants de PME industrielles syriennes, montre que les données comptables de gestion constituent un point d'ancrage obligé pour tous les dirigeants, y compris ceux des entreprises les plus petites, mais que ces données sont rarement très complexes et restent par là-même spécifiques à ce type d'organisation. Elle révèle ensuite l'existence de contingences structurelles et comportementales.

Mots clés : données comptables de gestion, dirigeants de PME, contingences.

Abstract: The study, carried out by face-to-face directive interviews with 92 managers of syrian industrial SMEs, highlights that the accounting information management is a mandatory anchor for all managers, including those of smaller companies, but the accounting information systems in SMEs are rarely complex and are, thereby, specific. The study then reveals the existence of structural and behavioural contingencies.

Keywords: Management accounting data, SME managers, contingency factors

\section{Introduction}

La littérature décrit le système de gestion du dirigeant de PME comme spécifique (Marchesnay, 1992 ; Julien, 1997). Pour prendre leurs décisions, les dirigeants de PME s'appuieraient plutôt sur les sources d'informations externes que sur les sources internes, sur les sources informelles que sur les sources formelles, sur les sources non-financières que sur les sources financières (Bescos et Mendoza, 1998 ; Beldi et Cheffi, 2005 ; Mintzberg, 2006 ; Vallerand et al., 2008). En tant que sources formelles, internes et financières, les données comptables de gestion pourraient s'avérer mal adaptées aux besoins des dirigeants de PME et être reléguées au second plan par ceux-ci.

Dans quelle mesure les données comptables de gestion sont-elles produites et utilisées par les dirigeants de PME ? Le bilan des recherches empiriques réalisées sur le thème du système d'information comptable (SIC) en PME aboutit à des conclusions contradictoires. Plusieurs d'entre elles confirment que les dirigeants des entreprises les plus petites disposent de SIC peu complexes et peu formalisés, qu'ils utilisent peu à des fins de gestion (Nayak et Greenfield, 1994 ; Colot et Michel, 1996 ; Davila, 2005 ; Zawadski, 2011). Mais d'autres soulignent que cette vision ne correspond pas à la réalité de l'ensemble des pratiques en PME. Ils montrent qu'une majorité de dirigeants de PME, même parmi les plus petites, dispose de SIC composés de données comptables de gestion qu'ils utilisent pour prendre leurs décisions, et relèvent que la complexité des SIC varie d'une PME à l'autre (Rowe et al., 1994 ; Lavigne, 2002 ; Affès et Chabchoub, 2007).

L'objet de ce travail consiste : 1- à proposer une description des SIC en PME afin de déterminer dans quelle mesure et sous quelles conditions les données comptables de gestion sont produites et utilisées ; 2- à identifier les facteurs de contingence susceptibles d'influencer leur complexité.

Cette problématique est abordée dans un contexte particulier, celui de la Syrie. L'ouverture de l'économie de ce pays sur le monde extérieur est très récente puisqu'elle date de l'an 2000 et reste fragile, les évènements politiques survenus en 2012 le montrent. La période précédente (1960-2000) était marquée par une économie socialiste, planifiée, résolument tournée vers une production autarcique visant l'autosuffisance (Aita, 2007). Le contexte économique, financier et géopolitique ne favorisait pas le développement du secteur privé. Depuis une dizaine d'années, la Syrie est passée progressivement d'une économie fortement contrôlée par l'Etat à une économie de marché. Plusieurs réformes ont visé à faciliter les investissements domestiques et étrangers pour compenser la baisse programmée des recettes pétrolières. La décision en juin 2005 de mettre en place une économie sociale de marché, l'arrivée de nouveaux investisseurs, l'accord de libre-échange avec les 
pays arabes entré en vigueur le $1^{\text {er }}$ janvier 2005 et la Turquie en 2007, et la libération progressive du commerce extérieur, ont forcé les entreprises syriennes, habituées au protectionnisme, à se moderniser et à changer leurs modes de fonctionnement.

S'appuyant sur la théorie de la contingence dans le cadre de la théorie de la spécificité (Marchesnay, 1992; Julien, 1997; Torrès, 2004), notre étude constitue une tentative de caractérisation des SIC des dirigeants de PME afin de déterminer dans quelle mesure ceux-ci s'avèrent spécifiques à la PME et d'identification des facteurs de contingence susceptibles d'impacter leur degré de complexité.

\section{Cadre théorique et méthodologique}

La première partie de cet article est dédiée à la présentation du cadre théorique sur lequel nous nous basons pour décrire les propriétés du SIC et identifier ses déterminants, et de la méthodologie mise en œuvre.

\subsection{Définitions et hypothèses de la recherche}

Avant de présenter le modèle de recherche, nous définissons la PME et le SIC afin de fixer les limites de notre champ d'investigation.

\subsubsection{Une définition de la PME}

Nous entendons par PME, toute entreprise juridiquement indépendante disposant d'un effectif compris entre 10 et 250 salariés. D'une manière très classique (Marchesnay, 2003), les critères retenus concernent d'une part le nombre de salariés et d'autre part l'indépendance de l'entreprise en termes de capital. L'effectif est un critère nécessaire mais insuffisant pour définir la PME. Les PME dépendantes d'entreprises plus grandes se voient en effet souvent proposer des procédures qui modifient leur système de gestion (Marchesnay, 2003). Sont donc exclues de notre champ de recherche les très petites entreprises de moins de 10 salariés, les entreprises de plus de 250 salariés, ainsi que les filiales, succursales ou divisions d'entreprises plus importantes.

Nous nous focalisons en outre sur les PME industrielles. Ce choix se justifie par leur importance pour l'économie syrienne. D'après le ministère de l'industrie syrien, 99,6\% des entreprises industrielles privées ont un effectif inférieur à 250 salariés. Le secteur industriel privé emploie $79,4 \%$ de la main d'œuvre industrielle et assure $78 \%$ des exportations industrielles du pays.

\subsubsection{Une approche élargie du SIC}

Trois dimensions ont été retenues pour caractériser le SIC :

- Une dimension organisationnelle : les caractéristiques de l'unité formellement chargée de produire et diffuser les données comptables. Saboly (1994) souligne que «les données comptables sont produites dans et par une organisation comptable et la qualité du produit comptable dépend de certaines caractéristiques de cette organisation ». Nous avons ainsi intégré une dimension organisationnelle à la définition du SIC afin de décrire leurs propriétés de manière plus globale et complète.

- Une dimension relative aux données comptables produites : les données comptables obligatoires et facultatives, historiques et prévisionnelles relatives à la Comptabilité Générale (CG), au Système de calcul de Coûts (SC), au Système Budgétaire (SB) et au Système de Suivi (SS) disponibles dans la PME.

- Une dimension relative aux données comptables utilisées par le dirigeant de PME. A l'instar de Malo (1989), nous pensons que «le domaine de la recherche ne peut être limité à l'offre ou à la production des données comptables. Leur utilisation doit aussi être étudiée ne serait-ce que pour préciser que produire ou encore comment produire». C'est pourquoi il convient de chercher dans quelle mesure et sous quelles conditions les dirigeants de PME sont susceptibles de «mettre en acte », selon l'expression de Weick (1995), les données de la CG, du SC, du SB et du SS. Du degré d'utilisation des données en question dépend leur pertinence, et réciproquement. Si le dirigeant les utilise, cela signifie qu'elles sont pertinentes à ses yeux.

Figure 1 : Les composantes du SIC du dirigeant de PME

\begin{tabular}{|c|c|c|}
\hline \multicolumn{3}{|c|}{ Système d'Information Comptable (SIC) } \\
\hline Organisation du service comptable & Production des données comptables & Utilisation des informations comptables \\
\hline
\end{tabular}


Le SIC peut ainsi être défini comme un ensemble organisé de structure, moyens et acteurs permettant de produire des données comptables (obligatoires et facultatives, historiques et prévisionnelles) utilisées par les dirigeants de PME pour gérer leur entreprise.

Notre étude retient comme critère de caractérisation du SIC, son degré de complexité. Cette prise en considération présente plusieurs intérêts à la fois théoriques et méthodologiques.

D'un point de vue théorique, la notion de complexité a fait irruption dans les travaux de recherche en sciences comptables à la fin des années 60. Bon nombre des chercheurs qui se sont intéressés aux SIC ont utilisé ce critère pour caractériser l'objet de leur recherche (Bergeron, 1996 ; Germain, 2004 ; Tillema, 2005 ; Jänkälä, 2007 ; Al-Omiri et Drury, 2007 ; Abdel-Kader et Luther, 2008). L'usage de ce critère nous permettra de confronter nos résultats à la théorie de la spécificité des PME qui souligne que les caractéristiques de ce type d'organisations devraient conduire leurs dirigeants à mettre en place des SIC peu complexes (Marchesnay, 1992 ; Julien, 1997).

D'un point de vue méthodologique, l'usage du critère de complexité présente plusieurs avantages :

- c'est en premier lieu un indicateur particulièrement bien adapté pour construire une typologie ou pour établir un classement sur un continuum : des SIC faiblement complexes aux SIC fortement complexes,

- ensuite, de par son caractère synthétique, il peut aisément servir de support dans le cadre d'analyses statistiques d'associations entre variables. Cet aspect apparaît particulièrement intéressant pour la réalisation d'une analyse contingente consistant à repérer l'existence de relations entre les SIC et les facteurs de contingence considérés par l'analyste.

\subsubsection{Les déterminants de la complexité du SIC des PME : le modèle de recherche}

Le SIC a trouvé naissance et s'est développé dans les organisations tayloriennes. C'est pourquoi il a longtemps, et parfois implicitement, été considéré par les chercheurs comme globalement et universellement pertinent et cohérent. Un passage de la conception universaliste à la compréhension contingente des SIC s'est toutefois imposé dans la littérature au début des années 1970. Il a conduit les chercheurs du domaine à s'interroger sur la capacité des SIC à se différencier selon les spécificités de la structure et du contexte où ils sont développés. On peut sur ce point citer les travaux précurseurs de Khandwalla (1972), Sathe (1975) ou de Bruns et Waterhouse (1975).

Plusieurs recherches ont ainsi démontré l'existence de liens entre les caractéristiques structurelles et contextuelles de l'entreprise et son SIC (Chenhall, 2003 ; Bescos et al., 2004 ; Jänkälä, 2007 ; AlOmiri et Drury, 2007 ; Abdel Kader et Luther, 2008). Ces auteurs retiennent la théorie de la contingence structurelle comme assise théorique. Ils posent le SIC comme éminemment adaptable aux différents types d'organisations.

L'analyse des travaux réalisés sur ce thème nous permet de retenir six facteurs de contingence pour tenter d'expliquer le degré de complexité des SIC en PME : la taille de l'entreprise (Chapellier, 1996 ; Nobre, 2001 ; Chenhall, 2003), la structure de propriété (Germain, 2001 ; Lavigne, 2002 ; Lavigne et Saint-Pierre, 2002 ; Saint-Pierre et Bahri, 2003 ; Affès et Chabchoub, 2007 ; Cheffi et Nekhili, 2011), l'endettement (Coker et Hayes, 1992 ; Lavigne et Saint-Pierre, 2002), l'exportation (Touchais, 1998 ; Lavigne, 2002; Vallerand et al., 2008 ), le type de stratégie (Chenhall et Langfield-Smith, 1998 ; Chenhall, 2003 ; Jänkäkä, 2007 ; Sandrino, 2007 ; Boulianne, 2007), l'incertitude perçue de l'environnement (Bergeron, 1996; Teller, 1999 ; Haldma et Lääts, 2002 ; Lavigne, 2002 ; Germain, 2004 ; Abdel-Kader et Luther, 2008).

La première hypothèse générale sera donc la suivante :

Ha : il existe une relation significative entre la complexité du SIC des PME et certaines caractéristiques structurelles et contextuelles de l'entreprise.

Elle se décompose en 6 sous-hypothèses :

Ha1 : Le degré de complexité du SIC augmente quand la taille des PME augmente

Ha2 : Le degré de complexité du SIC augmente lorsqu'un ou plusieurs actionnaires non apparentés détiennent une partie du capital

Ha3 : Les PME endettées disposent d'un SIC plus complexe que les PME non endettées

Ha4: Les PME exportatrices disposent d'un SIC plus complexe que les PME non exportatrices 
Ha5 : Les PME qui mettent en place une stratégie de type «prospecteur» disposent d'un SIC plus complexe que les PME qui mettent en place une stratégie de type « défenseur»
Ha6 : Les PME évoluant dans un environnement perçu comme dynamique et incertain disposent d'un SIC plus complexe que les PME évoluant dans un environnement perçu comme simple et stable

Mais la théorie de la contingence structurelle néglige l'influence possible des acteurs. D'autres auteurs (Chapellier, 1996 ; Bergeron, 1996 ; Lavigne, 2002 ; Flacke et Segbers, 2005 ; Nyengue Edimo, 2006; Affès et Chabchoub, 2007 ; Santin et Van Caillie, 2008) ont proposé pour cette raison d'élargir le cadre de recherche sur les systèmes d'information comptables à la théorie de la contingence comportementale. Dans cette perspective, les caractéristiques individuelles de chaque acteur viennent modérer l'aspect normatif des variables structurelles. Un individu peut donc être actif par rapport à la situation et non contraint par elle. Ainsi, et plus précisément, les caractéristiques organisationnelles et de contexte peuvent certes, dans une certaine mesure, limiter et déterminer les choix de l'acteur, mais ce dernier n'en reste pas moins libre et doué d'intentionnalité. Il dispose d'une marge de liberté (Crozier et Friedberg, 1977). Comme le souligne Nobre (1999) : «après avoir privilégié les aspects purement techniques, le contrôle de gestion intègre des éléments pour tenir compte des comportements des acteurs dans l'organisation ».

Les facteurs de contingence comportementaux retenus par les auteurs tournent le plus souvent autour de l'acteur central de la PME : le dirigeant. L'ensemble de la littérature s'accorde en effet pour souligner que le profil du dirigeant (compétence, histoire, culture, famille...) joue un rôle très particulier qui fait la spécificité de ce type d'organisation: " comprendre la PME, c'est tout d'abord découvrir le profil et percer les motivations de son propriétaire-dirigeant » (Raymond et $a l ., 2004)$. Ses caractéristiques pourraient avoir un impact significatif sur la complexité du SIC et à ce titre, méritent d'être analysées. A la lecture des travaux réalisés, les caractéristiques descriptives du dirigeant retenues dans cette étude sont : son degré de participation au capital (Lavigne, 2002 ; Ngongang, 2007), son niveau de formation (Saboly, 1994 ; Chapellier, 2003), son type de formation et son expérience (Chapellier, 2003).

La littérature montre enfin l'importance en PME de l'accompagnement du dirigeant (Couteret, 2010). Divers auteurs montrent notamment que les professionnels comptables internes et externes peuvent jouer un rôle important et influencer la complexité des SIC (Marriott et Marriott, 2000 ; Nandan, 2010). C'est pourquoi nous avons intégré au modèle des variables relatives à la mission du comptable interne (Saboly, 1994) et au degré d'implication de l'expert-comptable externe (Chapellier, 2003, Nandan, 2010, Barlette et Jaouen, 2012).

Notre recherche testera l'hypothèse générale suivante :

\section{Hb : il existe une relation significative entre la complexité du SIC des PME et certaines caractéristiques des acteurs de l'entreprise.}

Cette deuxième hypothèse générale se décompose en 6 sous-hypothèses :

Hb1 : Les PME pilotées par un dirigeant non actionnaire ou actionnaire minoritaire disposent d'un SIC plus complexe que les PME pilotées par un dirigeant actionnaire majoritaire

Hb3 : Le degré de complexité du SIC augmente lorsque le dirigeant dispose d'un diplôme de type comptable ou gestionnaire

Hb5 : Il existe une relation significative entre la mission du comptable interne et le degré de complexité du SIC
Hb2 : Le degré de complexité du SIC augmente avec le niveau de formation du dirigeant

Hb4 : Les PME pilotées par un dirigeant peu expérimenté disposent d'un SIC plus complexe que les PME pilotées par un dirigeant très expérimenté

Hb6 : Le degré de complexité du SIC augmente avec le degré d'implication de l'expert-comptable externe en matière de gestion

L'hypothèse dominante de la littérature est donc que les caractéristiques des SIC utilisés par les dirigeants de PME varient en fonction de variables structurelles et/ou comportementales. Mais d'autres travaux nuancent cette affirmation en montrant qu'en tant que sources formelles, internes et financières, les données comptables en PME sont plutôt rudimentaires et peu utilisées, car mal adaptées aux besoins des dirigeants (Beldi et Cheffi, 2005 ; Mintzberg, 2006 ; Vallerand et al., 2008). Ils soulignent que les pratiques se révèlent en la matière assez homogènes, et dépassent de ce point de vue les contingences (Nayak et Greenfield, 1994 ; Colot et Michel, 1996 ; Davila, 2005 ; Zawadski, 2011). Il ressort finalement de la littérature une relative indétermination quant aux 
caractéristiques des SIC en PME. L'étude de cette question nécessite un éclairage complémentaire que nous proposons de réaliser en nous appuyant sur le cadre de recherche suivant:

Figure 2 : Le cadre de la recherche

Facteurs structurels et contextuels

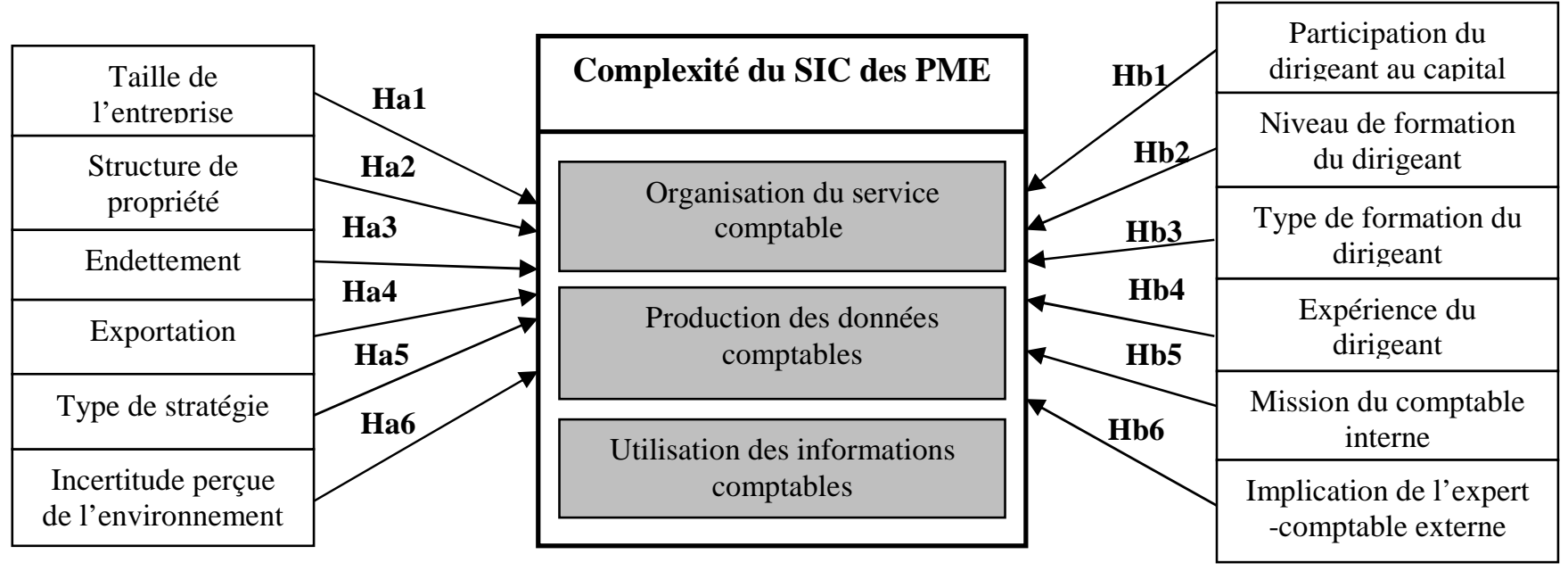

\subsection{Méthodologie de la recherche}

La méthode de cueillette des données, l'opérationnalisation des variables, les outils statistiques utilisés pour analyser les données et les caractéristiques de l'échantillon, sont successivement présentés.

\subsubsection{La méthode de cueillette des données}

L'enquête s'est déroulée en deux temps. Tout d'abord une enquête préliminaire a été conduite par administration, par entretiens directs, d'une première version du questionnaire. Ce pré-test, réalisé aux mois de janvier et février 2008 auprès de 18 dirigeants de PME industrielles de la ville d'Alep ${ }^{2}$, a permis une mise à l'épreuve de la forme des questions, de leur ordonnancement, une revue du vocabulaire courant, une vérification de la compréhension des répondants et de la pertinence des modalités de réponse proposées.

La deuxième phase de l'étude s'est déroulée au cours de l'été 2008. Les PME de l'échantillon ont été choisies par tirage aléatoire dans une base de données disponible à la Chambre de l'Industrie d'Alep : 218 dirigeants de PME industrielles juridiquement indépendantes, disposant d'un effectif compris entre 10 et 250 salariés ont été contactés par téléphone. La seconde version du questionnaire a été administrée par entretiens directs auprès de 92 dirigeants (soit un taux d'acceptation de 42\%). La durée minimum de l'entretien a été de 30 minutes, la durée maximale de 3 heures 30 et la durée moyenne de 45 minutes.

Comme l'objectif consistait à repérer des relations entre un grand nombre d'objets et à les décrire dans un modèle qui soit généralisable, nous avons eu recours à une méthode de recherche quantitative. Les statistiques descriptives réalisées permettent de mette en exergue les caractéristiques des organisations et des acteurs observés, et de synthétiser les données recueillies pour dresser des photographies. Au-delà d'une description immédiate des pratiques, le recours aux statistiques explicatives permet d'analyser les liens entre les concepts, c'est-à-dire les relations entre la variable à expliquer - la complexité du SIC - et les variables explicatives - les contingences -.

Le mode d'administration du questionnaire par entretien direct a été retenu car il autorise un recueil d'informations plus complet, plus détaillé et de meilleure qualité que l'enquête postale ou électronique (Bergeron, 1996 ; Chapellier, 1996 ; Evrard et al., 2009). Il a permis en effet de poser des questions délicates, longues, variées et d'apporter certaines précisions utiles au répondant et à la validité de l'étude grâce à la possibilité de reformuler, de clarifier et d'expliquer si nécessaire aux répondants les questions difficiles ou mal comprises (Thiétart, 2007). Administrer ces questionnaires en face à face a donc permis d'interroger à la fois la complexité de forme et la complexité de sens des données comptables. De plus, la confidentialité qui entoure la comptabilité

\footnotetext{
${ }^{2}$ Alep est la plus grande ville de Syrie. Elle est considérée comme la capitale industrielle et commerciale du pays.
} 
en PME aurait probablement conduit la plupart des dirigeants à refuser de répondre à un questionnaire reçu par voie postale ou électronique. Le fait de pouvoir expliquer et justifier en face à face l'objet de l'étude a généré de la confiance. Il a rendu possible la réalisation de cette recherche.

\subsubsection{L'opérationnalisation des variables}

Le questionnaire était structuré en thématique (les caractéristiques de l'entreprise et de son environnement, les caractéristiques du SIC et les caractéristiques du dirigeant et des professionnels comptables) et contenait à la fois des questions fermées avec échelles graduées et des questions ouvertes qui permettent de donner des réponses plus vastes.

Le construit permettant de mesurer la complexité du SIC s'appuie sur six recherches antérieures majeures : Saboly (1994), Chapellier (1996), Bergeron (1996), Lavigne (2002), Germain (2004) et Nyengue Edimo (2006). Mais les échelles ont été élaborées spécifiquement pour les besoins de l'enquête. Le tableau 1 présente la manière dont les variables relatives au SIC ont été opérationnalisées.

Tableau 1 : Mesure de la complexité du SIC

\begin{tabular}{|c|c|c|}
\hline Dimensions du SIC & Variables & Items de mesure (sur une échelle de cinq points) \\
\hline \multirow{4}{*}{$\begin{array}{l}\text { Organisation du } \\
\text { service comptable } \\
\quad \text { (sur } 20 \text { points) }\end{array}$} & $\begin{array}{l}\text { 1) La morphologie du service } \\
\text { comptable }\end{array}$ & $\begin{array}{l}\text { Effectif et nombre de niveaux hiérarchiques du service } \\
\text { comptable }\end{array}$ \\
\hline & $\begin{array}{l}\text { 2) Le niveau de spécialisation } \\
\text { du service comptable }\end{array}$ & $\begin{array}{l}\text { Nombre et type d'activités comptables réalisées au sein du } \\
\text { service }\end{array}$ \\
\hline & 3) Le degré d'informatisation & $\begin{array}{l}\text { Nombre d'ordinateurs utilisés ; nombre et type d'applications } \\
\text { informatiques dédiées à l'élaboration des données comptables }\end{array}$ \\
\hline & $\begin{array}{l}\text { 4) Formation du comptable } \\
\text { interne }\end{array}$ & $\begin{array}{l}\text { Niveau et type de formation du comptable interne responsable } \\
\text { du service }\end{array}$ \\
\hline \multirow{4}{*}{$\begin{array}{l}\text { Production des } \\
\text { données comptables } \\
\quad \text { (sur } 20 \text { points })\end{array}$} & 1) Le délai & \multirow{4}{*}{$\begin{array}{l}\text { Délai, fréquence, diversité et degré de détail des données de } \\
\text { Comptabilité Générale (CG), du Système de calcul de Coûts } \\
\text { (SC), du Système Budgétaire (SB) et du Système de Suivi (SS) }\end{array}$} \\
\hline & 2) La fréquence & \\
\hline & 3) La diversité & \\
\hline & 4) Le détail & \\
\hline \multirow{4}{*}{$\begin{array}{l}\text { Utilisation des } \\
\text { informations } \\
\text { comptables } \\
\text { (sur } 20 \text { points) }\end{array}$} & 1) La fréquence & Fréquence de l'utilisation des informations de CG, SC, SB et SS \\
\hline & 2) La durée & $\begin{array}{l}\text { Durée moyenne hebdomadaire de l'utilisation de l'information } \\
\text { comptable }\end{array}$ \\
\hline & 3) L’intensité & \multirow{2}{*}{$\begin{array}{l}14 \text { items représentant des situations d'utilisation potentielle de } \\
\text { l'information comptable sur une échelle de } 1 \text { à } 5\end{array}$} \\
\hline & 4) La diversité & \\
\hline
\end{tabular}

Chacune des 3 dimensions est appréciée par 4 variables mesurées sur une échelle de 5 points (soit un maximum de 20 points par dimension et un score maximal de complexité de 60 points).

Les coefficients de corrélation de Pearson calculés entre les items, variables et scores de complexité, garantissent la validité de notre construit : les items utilisés pour mesurer la complexité du SIC convergent.

Le coefficient Alpha de Cronbach des 12 variables caractérisant le SIC est à 0,94. L'instrument de mesure élaboré a donc une bonne cohérence interne et permet de mesurer fidèlement la complexité du SIC. La somme de ces variables peut être prise comme une mesure synthétique du degré de complexité du SIC des PME.

Le tableau 2 présente la manière dont les variables explicatives ont été opérationnalisées.

Tableau 2 : Mesures des variables explicatives

\begin{tabular}{|l|l|}
\hline \multicolumn{1}{|c|}{ Variables } & \multicolumn{1}{|c|}{ Mesure } \\
\hline Taille & Nombre de salariés \\
\hline Structure de propriété & $\begin{array}{l}\text { Un seul actionnaire, plusieurs actionnaires apparentés, plusieurs actionnaires non } \\
\text { apparentés }\end{array}$ \\
\hline Exportation & Exportatrice / non exportatrice \\
\hline Endettement & Endettée / non endettée \\
\hline $\begin{array}{l}\text { Incertitude perçue de } \\
\text { l'environnement }\end{array}$ & $\begin{array}{l}\text { Cinq items sur une échelle de Likert à 5 points portant sur le dynamisme de } \\
\text { l'environnement économique et technologique, la prévisibilité de l'activité des } \\
\text { concurrents et des goûts des clients, et la fréquence de révision de la politique marketing }\end{array}$ \\
\hline Stratégie & $\begin{array}{l}\text { Six items mesurés sur une échelle de Likert à 5 points mesurant l'attitude du dirigeant } \\
\text { face à six actions reliées aux produits et aux marchés }\end{array}$ \\
\hline
\end{tabular}




\begin{tabular}{|l|l|}
\hline Participation du dirigeant au capital & Actionnaire majoritaire / actionnaire minoritaire / non actionnaire \\
\hline Expérience du dirigeant & Nombre d'années passées à la tête d'une entreprise \\
\hline Niveau de formation du dirigeant & Inférieur au Bac / Bac et Bac+2 / Maîtrise / 3 3è $^{\text {cycle }}$ \\
\hline Type de formation du dirigeant & Gestionnaire / non gestionnaire \\
\hline Mission du comptable interne & Pas de comptable interne / Aide-comptable / Comptable / Contrôleur de gestion \\
\hline $\begin{array}{l}\text { Implication de l'expert-comptable } \\
\text { externe en matière de gestion }\end{array}$ & $\begin{array}{l}\text { Pas d'expert-comptable externe / Implication faible en matière de gestion / Implication } \\
\text { forte en matière de gestion }\end{array}$ \\
\hline
\end{tabular}

\subsubsection{La méthode d'analyse des données}

Le modèle statistique retenu pour tester les hypothèses est celui de la régression linéaire multiple par la méthode des Moindres Carrés Ordinaires (MCO). La régression est réalisée par la méthode de régression pas à pas «stepwise $»^{3}$. Cette méthode est recommandée lorsque le chercheur dispose d'un grand nombre de variables explicatives (Jolibert et Jourdan, 2006 ; Malhotra, 2007). Tel est le cas dans ce travail.

La régression linéaire multiple teste la relation entre une variable dépendante quantitative et plusieurs variables indépendantes quantitatives. Afin de répondre à cette exigence, vu que les variables de notre étude sont mixtes (quantitatives et qualitatives), une transformation préalable des variables qualitatives est indispensable :

- Certaines variables qualitatives ayant plus de deux modalités ont été transformées en variable qualitative dichotomique à deux modalités de réponse (0/1). C'est le cas des variables « structure de la propriété » (familiale / non familiale), «participation au capital » (majoritaire / non majoritaire) et «niveau de formation du dirigeant » (inférieur à Bac+4 / supérieur ou égal à $\mathrm{Bac}+4$ ).

- Les variables «mission du comptable interne » et «implication de l'expert-comptable externe » n'ont pu être réduites à deux modalités. Nous avons eu recours au codage de booléens préconisé dans cette situation par Jolibert et Jourdan (2006), qui consiste à transformer chaque variable qualitative à plus de deux modalités en $\mathrm{N}-1$ variables dichotomiques muettes $(\mathrm{N}=$ nombre de modalités).

\subsubsection{Les caractéristiques du terrain d'observation}

Les caractéristiques générales des PME de l'échantillon et de leurs dirigeants sont présentées dans les tableaux 3 et 4 .

Tableau 3 : Les entreprises de l'échantillon

\begin{tabular}{|c|c|c|}
\hline Secteur industriel & $\mathbf{N}$ & $\%$ \\
\hline Textile \& habillement & 46 & $50 \%$ \\
\hline Ingénierie & 12 & $13 \%$ \\
\hline Agro-alimentaire & 20 & $21,7 \%$ \\
\hline Chimie \& plastique & 14 & $15,3 \%$ \\
\hline \multicolumn{3}{|l|}{ Taille de l'entreprise } \\
\hline 10 à 39 salariés & 33 & $35,9 \%$ \\
\hline 40 à 74 salariés & 25 & $27,1 \%$ \\
\hline 75 à 149 salariés & 18 & $19,6 \%$ \\
\hline 150 à 250 salariés & 16 & $17,4 \%$ \\
\hline \multicolumn{3}{|l|}{ Âge de l'entreprise } \\
\hline 1 à 5 ans & 18 & $19,6 \%$ \\
\hline 6 à 10 ans & 25 & $27,2 \%$ \\
\hline 11 à 20 ans & 21 & $22,8 \%$ \\
\hline Plus de 20 ans & 28 & $30,4 \%$ \\
\hline Total & 92 & $100 \%$ \\
\hline
\end{tabular}

Tableau 4 : Les dirigeants de l'échantillon

\begin{tabular}{|l|l|l|}
\hline \multicolumn{1}{|c|}{$\begin{array}{c}\text { Participation du dirigeant au } \\
\text { capital }\end{array}$} & N & \multicolumn{1}{c|}{$\%$} \\
\hline Actionnaire unique & 21 & $22,8 \%$ \\
\hline Actionnaire majoritaire & 34 & $37 \%$ \\
\hline Actionnaire minoritaire & 33 & $35,9 \%$ \\
\hline Non actionnaire & 4 & $4,3 \%$ \\
\hline \multicolumn{1}{|c|}{ Age du dirigeant } & & \\
\hline Moins de 40 ans & 32 & $34,8 \%$ \\
\hline 40 à 49 ans & 27 & $29,3 \%$ \\
\hline 50 ans et plus & 33 & $35,9 \%$ \\
\hline \multicolumn{1}{|c|}{ Formation du dirigeant } & & \\
\hline Inférieur au Bac & 12 & $13 \%$ \\
\hline Bac et Bac+2 & 30 & $32,6 \%$ \\
\hline Maîtrise & 43 & $46,8 \%$ \\
\hline 3ème cycle & 7 & $7,6 \%$ \\
\hline Total & $\mathbf{9 2}$ & $\mathbf{1 0 0 \%}$ \\
\hline
\end{tabular}

La moitié des entreprises rencontrées proviennent du secteur textile et habillement. Ce chiffre reflète l'importance et la surreprésentation de cette industrie en Syrie en général et à Alep en particulier. La plupart des entreprises de notre échantillon sont de petite taille $63 \%$ ont moins de 75

\footnotetext{
${ }^{3}$ L'examen de la tolérance et du facteur d'inflation de la variance (VIF), et l'examen visuel de l'histogramme de la distribution des résidus et du graphique entre les résidus normalisés et les valeurs prévues normalisées de la variable dépendante, permettent de confirmer l'absence de problème de multicolinéarité entre les variables du modèle. Ils montrent que les résidus sont normalement distribués et que l'hypothèse de la variance constante de ces résidus est statistiquement respectée.
} 
salariés) et assez jeunes (près de 70\% ont été créées dans les 20 dernières années). 95,7\% des dirigeants rencontrés participent au capital de l'entreprise et $22,8 \%$ détiennent seuls la totalité du capital. Plus d'un tiers $(34,8 \%)$ a moins de 40 ans et plus d'un sur deux $(54,3 \%)$ a un diplôme de niveau égal ou supérieur à bac+4.

\section{Résultats de la recherche}

Les résultats de l'analyse des SIC en PME d'une part, et des facteurs explicatifs de leur degré de complexité d'autre part, sont successivement présentés.

\subsection{Des données comptables spécifiques qui constituent un point d'ancrage obligé pour tous les dirigeants de PME}

Les SIC sont présents dans toutes les PME observées mais ils sont rarement complexes. La moyenne de complexité est de 30,89/60, l'écart-type de 11,42 et la médiane de 30,95. La note minimale est de 7,11/60 et la note maximale de 53,93/60 (tableau 5).

Tableau 5 : Statistiques descriptives du degré de complexité des SIC

\begin{tabular}{|c|c|c|c|c|c|}
\hline Dimensions & Moyenne & Médiane & Ecart-type & Min. & Max. \\
\hline Organisation du service comptable /20 & 11,77 & 12,5 & 4,86 & 1,0 & 19,5 \\
\hline Production des données comptables /20 & 8,50 & 7,75 & 4,13 & 0,75 & 17 \\
\hline Utilisation des informations comptables /20 & 10,62 & 10,64 & 3,47 & 2,29 & 18,34 \\
\hline Degré global de complexité du SIC /60 & $\mathbf{3 0 , 8 9}$ & $\mathbf{3 0 , 9 5}$ & $\mathbf{1 1 , 4 2}$ & $\mathbf{7 , 1 1}$ & $\mathbf{5 3 , 9 3}$ \\
\hline
\end{tabular}

Une présence presque généralisée d'un service comptable interne : $89 \%$ des entreprises observées (82 PME sur 92) déclarent l'existence d'un service comptable interne. Ce constat souligne une spécificité du contexte syrien. Une grande majorité des transactions en Syrie est réalisée en espèces. Le secret lié aux chiffres comptables est important. Les dirigeants préfèrent que la comptabilité, qui sera ensuite transmise à l'expert-comptable et à l'administration fiscale, soit dans un premier temps élaborée en interne.

Les PME disposent de responsables comptables spécialisés et qualifiés. Parmi les 82 comptables internes observés, 63 possèdent au moins une maîtrise et 72 disposent d'un diplôme de type comptable ou gestionnaire.

Notons enfin que le SIC des PME étudiées est largement informatisé puisque $87 \%$ des services comptables disposent d'un ou plusieurs ordinateurs et $85 \%$ utilisent un logiciel comptable. En revanche, seuls $30 \%$ ont informatisé la production des données comptables de gestion (le système de calcul de coûts, le système budgétaire ou le système de suivi).

Une tendance marquée à la production de données comptables de gestion : la vision réductrice du SIC, orientée vers la production des seuls documents obligatoires, dans des délais longs, et dans le seul but de satisfaire aux obligations imposées par l'administration fiscale, ne correspond pas à la réalité d'une grande majorité des entreprises observées. Aucun dirigeant ne se limite à la production des seuls documents obligatoires entendus au sens strict. Si effectivement quelques-uns disposent de SIC rudimentaires, d'autres élaborent à fréquence régulière des données comptables de gestion certes rarement très complexes mais relativement diversifiées et détaillées.

Les états financiers annuels obligatoires sont élaborés plutôt rapidement. De manière assez surprenante, 29,3\% affirment en disposer moins d'une semaine après la date de clôture et 48,9\% moins d'un mois après.

Des situations intermédiaires sont formalisées dans la plupart des PME rencontrées (77\%) : 35\% les élaborent mensuellement, $11 \%$ trimestriellement et $31 \%$ semestriellement. La moitié des dirigeants en dispose moins d'une semaine après la date de clôture et $42 \%$ dans le mois qui suit.

91,3\% des PME disposent d'un système de calcul de coûts. La méthode des coûts complets (seule ou associée à une autre méthode) est la plus fréquemment utilisée (68\%). Les coûts sont calculés mensuellement par 36\% des entreprises et trimestriellement par $23 \%$ d'entre elles. La production de ces données est très rapide : $63 \%$ des dirigeants affirment en disposer en moins d'une semaine.

Les données budgétaires sont en revanche moins présentes dans les PME de notre échantillon. Seules $40 \%$ déclarent élaborer formellement des budgets : $28 \%$ élaborent un budget des ventes, 
$39 \%$ un budget de production, 36\% un budget des approvisionnements, $29 \%$ un budget de trésorerie et seules $16 \%$ budgétisent leurs projets d'investissement. Ces budgets sont le plus souvent annuels (32\% des cas) ou semestriels ( $30 \%$ des cas).

Enfin, moins d'une entreprise sur quatre (24\%) dispose d'un système de suivi des budgets et calcule des écarts.

Une utilisation des données comptables généralisée mais à des degrés variables : Seuls 3,3\% des dirigeants déclarent ne jamais utiliser les informations comptables pour diriger leur entreprise. La plupart des chefs de PME utilisent ces données dans leurs processus décisionnels. Pour autant, ils ne le font que rarement de façon très intense, ce qui pourrait marquer une utilisation certes affirmée, mais selon des formes spécifiques de la PME. Les données en question sont en effet assez fréquemment utilisées pour connaître l'évolution de la trésorerie, le dû fournisseurs et le dû clients, pour suivre aussi l'évolution des performances, fixer les prix de vente, choisir de maintenir une activité, de l'abandonner, de la sous-traiter ou choisir de renouveler ou d'accroître le potentiel de production. Elles le sont en revanche assez peu pour prévoir la situation des semaines et des mois à venir, déterminer des objectifs par référence aux années passées, suivre la réalisation de ces objectifs, choisir un mode de financement ou gérer les emprunts en cours.

Notre étude montre ainsi que les données comptables prennent du sens aux yeux des dirigeants de PME, mais sous condition de simplification et d'adaptation. Les données comptables de gestion observées sont simplifiées et parfois d'apparence peu conforme aux indicateurs usuels en analyse financière et en contrôle de gestion. Mais, justement parce qu'elles sont moins détaillées, moins diversifiées et moins fréquemment élaborées que dans les entreprises de taille supérieure, ces données sont plus volontiers utilisées par les dirigeants de PME. Autrement dit, les dirigeants de PME ont recours à des données comptables simplifiées, ou plutôt adaptées, pour assurer leur intelligence comptable, c'est-à-dire leur capacité à distinguer les problèmes à partir de ces données comptables. En l'occurrence, la simplicité apparente de la forme n'occulte pas la complexité de sens. Elle en conditionne au contraire l'émergence. Au sein de la PME, les outils comptables très «sophistiqués » trouvent ainsi des limites de portée sans doute plus générale. Un SIC très développé et formalisé, qui véhiculerait implicitement la référence à une rationalité plus ou moins substantielle voire normative, ne ferait finalement pas sens pour les dirigeants de PME.

Ce constat conduit à considérer que les SIC en PME sont simplifiés et diversifiés, non pas parce que les données comptables sont jugées inutiles et peu pertinentes par les dirigeants, mais parce qu'elles sont adaptées à divers contextes, divers besoins et diverses compétences. Il apparaît alors pertinent de se demander dans quelle mesure les variables indépendantes de notre modèle de recherche expliquent cette diversité.

\subsection{Des tendances intermédiaires entre certains indices de déterminisme structurel et de déterminisme comportemental}

La seconde partie de la recherche consiste à tenter d'identifier les facteurs susceptibles d'influencer la complexité des SIC précédemment décrits. Le test des hypothèses a été réalisé en ayant recours à la régression linéaire par la méthode des Moindres Carrés Ordinaires (MCO) à l'aide de l'équation suivante :

DCOMSIC $_{i}=\beta_{0}+\beta_{1}\left(\right.$ TAIL $\left._{i}\right)+\beta_{2}\left(\boldsymbol{S T R P R O}_{i}\right)+\beta_{3}\left(\boldsymbol{E X P P}_{i}\right)+\beta_{4}\left(\boldsymbol{E N D}_{i}\right)+\beta_{5}\left(\boldsymbol{I P E}_{i}\right)+\beta_{6}\left(\boldsymbol{S T R A}_{i}\right)+\beta_{7}$ $\left(\right.$ PARCAP $\left._{i}\right)+\beta_{8}\left(\boldsymbol{E X P E R}_{i}\right)+\beta_{9}\left(\boldsymbol{N I V F O R}_{i}\right)+\beta_{10}\left(\boldsymbol{T Y P F O R}_{i}\right)+\beta_{11}\left(\boldsymbol{M I S 1}_{i}\right)+\beta_{12}\left(\boldsymbol{M I S 2}_{i}\right)+\beta_{13}\left(\boldsymbol{M I S 3}_{i}\right)$ $+\beta_{14}\left(\right.$IMPFAI $\left._{i}\right)+\beta_{15}\left(\right.$ IMPFOR $\left.\boldsymbol{R}_{i}\right)+\varepsilon_{i}$

DCOMSIC : degré de complexité du SIC, TAIL : taille de l'entreprise, STRPRO : structure de la propriété (familiale/ non familiale), EXP : exportation, END : endettement, IPE : incertitude perçue de l'environnement, STRA : stratégie, PARCAP : participation au capital, EXPER : expérience du dirigeant, NIVFOR : niveau de formation du dirigeant $(<$ Bac +4 ou $\geq$ Bac +4 ), TYPFOR : type de formation du dirigeant (gestionnaire ou non), MIS1 : mission d'aidecomptable, MIS2 : mission de comptable, MIS3 : mission de contrôleur de gestion, IMPFAI : implication faible en matière de gestion, IMPFOR : implication forte en matière de gestion, $\beta 0$ : la constante de la régression, $\beta_{1}, \beta_{2}, . ., \beta_{15}$ : paramètres à estimer (coefficients de régression standardisés), $\varepsilon:$ terme de l'erreur résiduelle.

Le tableau 6 présente les résultats de l'analyse de régression multiple appliquée au modèle de recherche. 
Tableau 6 : Résultats de la régression linéaire multiple

\begin{tabular}{|c|c|c|c|c|c|c|}
\hline Variables & Signe prédit & $\beta$ & $t$ & Sig. & Tolérance & VIF \\
\hline TAIL & + & 0,038 & $4,225^{* * * *}$ & $+^{* * * *}$ & 0,722 & 1,384 \\
\hline IPE & + & 0,701 & $3,849^{* * * *}$ & $++^{* * * * *}$ & 0,807 & 1,239 \\
\hline STRA & + & 0,576 & $3,989^{* * * *}$ & $++^{* * * *}$ & 0,807 & 1,239 \\
\hline NIVFOR & + & 3,417 & $2,81^{* *}$ & $+^{* * *}$ & 0,823 & 1,215 \\
\hline MIS2 & + & 6,551 & $3,792^{* * * *}$ & $++^{* * * *}$ & 0,618 & 1,619 \\
\hline MIS3 & + & 10,374 & $6,379^{* * * *}$ & $+^{* * * *}$ & 0,457 & 2,188 \\
\hline IMPFOR & + & 3,724 & $2,479^{*}$ & $+^{*}$ & 0,76 & 1,316 \\
\hline Constante & & $-1,054$ & & 0,3 & ,74) & \\
\hline EXP & + & \multirow{8}{*}{\multicolumn{5}{|c|}{ Variables exclues du modèle par la méthode pas à pas «Stepwise » }} \\
\hline END & + & & & & & \\
\hline STRPRO & + & & & & & \\
\hline PARCAP & - & & & & & \\
\hline EXPER & - & & & & & \\
\hline TYPFOR & + & & & & & \\
\hline MIS1 & - & & & & & \\
\hline IMPFAI & - & & & & & \\
\hline \multicolumn{2}{|l|}{$\mathbf{N}$} & \multicolumn{5}{|c|}{92} \\
\hline \multicolumn{2}{|l|}{$\mathbf{R}^{2}$} & \multicolumn{5}{|c|}{0,799} \\
\hline \multicolumn{2}{|l|}{$R^{2}$ ajusté } & \multicolumn{5}{|c|}{0,782} \\
\hline \multicolumn{2}{|c|}{ Coefficient $\mathbf{F}$ de Fisher $(\alpha)$} & \multicolumn{5}{|c|}{$47,57^{* * * *}$} \\
\hline
\end{tabular}

Il ressort de ce tableau que le modèle est significatif et a un pouvoir explicatif élevé. Les résultats statistiques confirment que 78,2\% de la variance du degré de complexité du SIC est expliquée par la régression. Autrement dit, le degré de complexité du SIC des PME syriennes observées est correctement expliqué par certaines variables de contingence structurelle et comportementale retenues dans notre modèle de recherche.

\subsubsection{Une influence marquée de certains facteurs d'ordre structurel et contextuel}

Les résultats de la régression montrent l'impact significatif de trois facteurs d'ordre structurel et contextuel : la taille de l'entreprise, le type de stratégie poursuivie et l'incertitude perçue de l'environnement.

La taille de l'entreprise est sans conteste un facteur déterminant de la complexité du SIC (Ha1). Nos résultats rejoignent sur ce point ceux des études antérieures (Nobre, 2001; Chenhall, 2003; Mintzberg, 2006; Abdel Kader et Luther, 2008). Ils montrent que l'accroissement de la taille entraine une complexification des outils comptables de gestion présents et utilisés dans l'entreprise.

Les tests effectués révèlent aussi l'existence d'un lien significatif entre le type de stratégie mis en place et la complexité du SIC (Ha5) confirmant ainsi les conclusions de Chenhall (2003) et de Boulianne (2007) qui suggèrent que certains outils comptables sont associés à certains types de stratégies. Les entreprises de notre échantillon disposent de SIC de complexité différente quand elles mettent en place des stratégies différentes : les dirigeants de type prospecteur disposent de SIC plus complexes que les dirigeants de type défenseur.

Les résultats de la régression montrent enfin l'effet significatif de l'incertitude perçue de l'environnement sur le degré de complexité du SIC (Ha6). Ce résultat concorde avec ceux de Germain (2004), de Haldma et Lääts (2002), et de Abdel-Kader et Luther (2008). Il indique que les PME évoluant dans un environnement perçu comme dynamique et incertain disposent d'un SIC plus complexe que celles évoluant dans un environnement perçu comme simple et stable.

En revanche, la méthode de régression pas à pas exclut trois facteurs structurels qui n'ont aucun lien significatif avec le degré de complexité du SIC: la structure de la propriété, l'endettement et l'exportation.

Aucune relation significative n'a en effet pu être établie entre la structure de propriété et la complexité du SIC (Ha2). Contrairement aux conclusions de Lavigne et Saint-Pierre (2002), et de Affès et Chabchoub (2007), la présence d'actionnaires ne faisant pas partie de la famille n'influence pas la complexité du SIC des PME observées. Les commentaires formulés par les dirigeants lors des entretiens nous permettent de suggérer l'hypothèse d'une spécificité culturelle : quelle que soit la structure de propriété, la PME syrienne est gérée avec une mentalité familiale. 
Aucun lien significatif n'apparaît entre le fait que l'entreprise ait des dettes et la complexité du SIC (Ha3). Lavigne (2002) suggère que la présence d'un créancier externe peut inciter les dirigeants à s'appuyer sur les outils de gestion pour prendre leurs décisions. Notre étude ne permet pas de valider cette hypothèse.

Les résultats révèlent enfin que le fait que la PME soit exportatrice n'influence pas le degré de complexité du SIC (Ha4). Le test bi-varié montre un impact significatif de cette variable sur la variable dépendante mais cet impact n'améliore pas de manière statistiquement significative la qualité globale de la régression $\left(\mathrm{R}^{2}\right)$.

\subsubsection{Une influence significative de certains facteurs d'ordre comportemental}

L'analyse révèle que les acteurs de la PME tiennent un rôle important dans le système d'influences qui s'exerce sur le degré de complexité du SIC.

\subsubsection{Un impact important de la formation du dirigeant}

Parmi les caractéristiques du dirigeant retenues dans notre modèle, seul son niveau de formation a un effet significatif sur la complexité du SIC $(\mathrm{Hb} 2)$ : les dirigeants de niveau Bac+4 ou plus, disposent d'un SIC plus complexe que ceux possédant un diplôme de niveau inférieur.

En revanche, les résultats montrent que la participation majoritaire du dirigeant au capital n'a pas d'impact sur la variable dépendante (Hb1) : le fait qu'il soit actionnaire majoritaire ou non n'affecte pas la complexité du SIC. Le mode de gouvernance de nombreuses PME syriennes est basé, quel que soit le degré de participation du dirigeant au capital, sur la collégialité. Cela explique pour partie que cette variable ne soit pas discriminante.

Aucun lien significatif n'apparaît entre le type de formation (gestionnaire ou non gestionnaire) et le degré de complexité du SIC (Hb3). Ce résultat ne concorde pas avec ceux des recherches antérieures qui, à l'exception de l'étude de Lavigne (2002), ont observé un lien étroit entre ces deux variables soulignant ainsi une sorte de normalisation des comportements par la formation (Chapellier, 2003). Tel n'est pas le cas dans notre étude. L'absence de relation peut s'expliquer par le faible pourcentage des dirigeants ayant reçu une formation gestionnaire (17,5\%). La comparaison des moyennes entre les deux groupes montre en effet que le premier (dirigeants ayant suivi une formation gestionnaire) dispose de SIC d'une complexité moyenne plus élevée que le second (35,31 contre 30,56), mais cette différence n'est statistiquement pas significative.

Les résultats de la régression relèvent enfin que l'expérience du dirigeant n'a pas d'influence significative sur le degré de complexité du SIC (Hb4). Nos résultats sont ici moins surprenants, les résultats des recherches réalisées jusqu'à présent étant sur ce point mitigés.

\subsubsection{Un impact important des caractéristiques des professionnels comptables}

\section{- L'influence significative de la mission du comptable interne}

La mission de $20 \%$ des 82 comptables internes de l'échantillon se limite à la tenue de la comptabilité, c'est-à-dire au travail préparatoire à l'établissement des documents annuels par l'expert-comptable (enregistrement et suivi des factures, états de rapprochements...), nous les avons qualifiés d'aides-comptables. La mission de $23 \%$ d'entre eux consiste à assurer la tenue de la comptabilité et, ponctuellement, à produire quelques données comptables à des fins de gestion, nous les avons qualifiés de comptables. Enfin, la mission de 57\% d'entre eux consiste à assurer la tenue de la comptabilité mais aussi, à produire de façon régulière des données comptables à des fins de gestion, nous les avons qualifiés de contrôleurs de gestion. Les résultats du modèle statistique montrent que la mission du comptable interne est fortement et significativement liée à la complexité du SIC (Hb5). Le degré de complexité du SIC des dirigeants assistés d'un comptable interne contrôleur de gestion est significativement élevé par rapport au degré de complexité du SIC des dirigeants assistés d'un comptable interne de type aide comptable ou comptable.

\section{- L'influence saillante de l'implication de l'expert-comptable externe}

La majorité des experts-comptables externes des PME de notre échantillon (69\%) intervient essentiellement pour préparer et certifier les documents comptables obligatoires. Seule une minorité (31\%) va au-delà et s'implique en matière de gestion (élaboration de budgets, calculs de coûts, 
conseils concernant le financement de l'entreprise, aide dans ses relations avec les créanciers, aide en matière de gestion des ressources humaines...). Les résultats du modèle statistique permettent d'établir un lien significatif entre l'implication de l'expert-comptable externe en matière de gestion et la complexité du SIC (Hb6) : les dirigeants assistés d'un expert-comptable externe fortement impliqué en matière de gestion, disposent d'un SIC plus complexe que ceux assistés d'un expertcomptable externe uniquement impliqué en matière fiscale ou n'ayant pas d'expert-comptable. Un accompagnement personnalisé et adapté, par un expert externe impliqué, impacte les pratiques du dirigeant de PME (Couteret et Audet, 2006).

Au final, l'analyse confirme 6 des douze hypothèses (figure 3) :

Figure 3 : Récapitulatif des résultats de l'analyse contingente

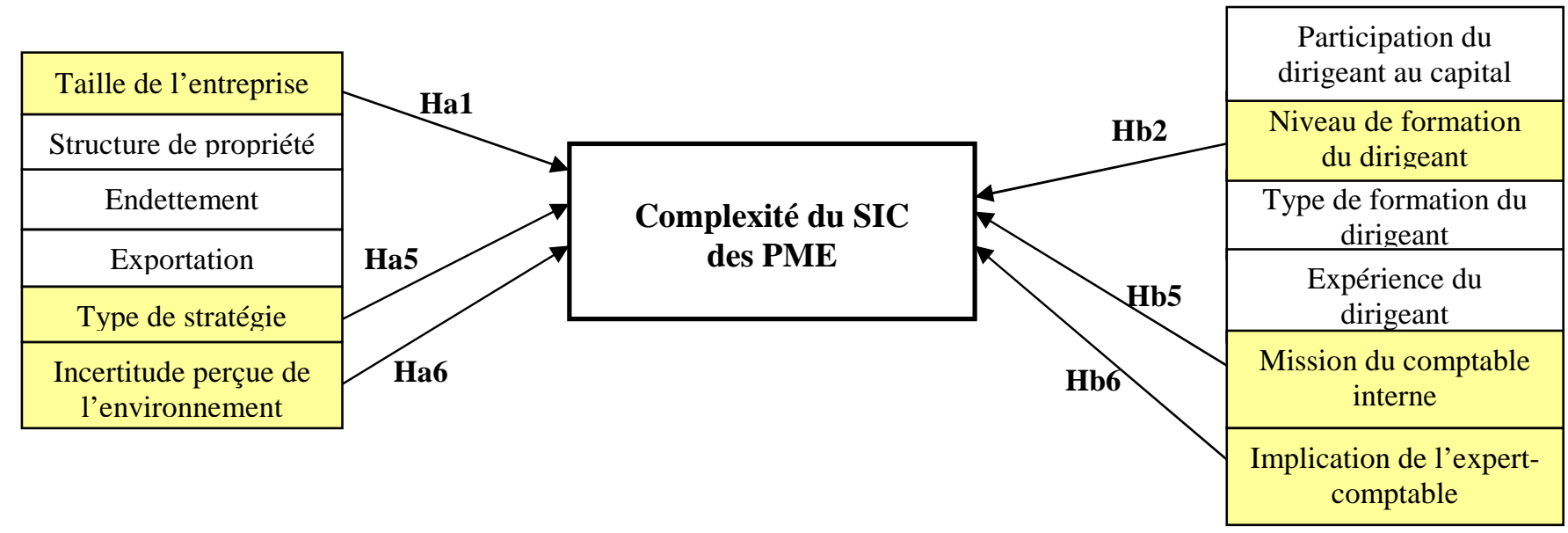

\section{Conclusion}

Les recherches empiriques réalisées jusqu'à présent sur le thème du SIC du dirigeant de PME aboutissent à des conclusions qui pourraient sembler contradictoires. Certaines montrent que les dirigeants des entreprises les plus petites disposent de SIC peu complexes et peu formalisés (Nayak et Greenfield, 1994 ; Colot et Michel, 1996 ; Davila, 2005 ; Zawadski, 2011). Mais d'autres soulignent qu'une importante majorité de dirigeants de PME, même parmi les plus petites, dispose de SIC relativement diversifiées qu'ils utilisent pour prendre leurs décisions (Rowe et al., 1994 ; Lavigne, 2002 ; Affès et Chabchoub, 2007).

Notre étude montre que ces deux pans de la littérature ne sont pas contradictoires :

- conformément aux affirmations des premiers, les SIC en PME sont rarement très complexes et restent par là-même spécifiques à ce type d'organisations,

- conformément aux affirmations des seconds, la plupart des dirigeants de PME disposent et utilisent une base minimale de données comptables de gestion dont la complexité varie en fonction du contexte, des besoins et des compétences.

L'article montre ainsi que les données comptables de gestion constituent un point d'ancrage obligé pour tous les dirigeants, y compris ceux des entreprises les plus petites. Le fait que ces données existent et que les dirigeants les utilisent, montre que le SIC en PME est simplifié, donc spécifique, non parce que ces données sont jugés inutiles ou peu pertinentes par les dirigeants, mais pour être adapté au contexte, aux besoins et aux compétences qui sont eux-mêmes spécifiques. Les données comptables prennent du sens aux yeux des dirigeants de PME, mais sous condition de simplification et d'adaptation. Les dirigeants de PME ont recours à des données comptables simplifiées et adaptées pour assurer leur intelligence comptable, c'est-à-dire leur capacité à distinguer les problèmes à partir de ces données. En l'occurrence, la simplicité apparente de la forme n'occulte pas la complexité de sens. Elle en conditionne au contraire l'émergence. Un système d'information comptable très développé et formalisé, qui véhiculerait implicitement la référence à une rationalité 
plus ou moins substantielle, ne ferait finalement pas sens pour le dirigeant de PME. Ignorer les particularismes et proposer aux dirigeants des outils standardisés serait inefficace (Couteret, 2010). Les SIC en PME doivent être adaptés au contexte et aux profils des acteurs. Cette adaptation relève d'un «fit» entre des facteurs de contingence structurels et contextuels, et des facteurs de contingence comportementaux : si certaines caractéristiques structurelles et contextuelles sont effectivement liées à la complexité du SIC (notamment la taille de la PME, le type de stratégie suivie et l'incertitude perçue de l'environnement), le dirigeant (notamment son niveau de formation) et les professionnels comptables internes et externes tiennent un rôle très important.

Ce travail montre donc qu'il n'existe pas de norme dictant le degré adéquat de recours aux données comptables. L'enjeu pour le dirigeant est de trouver le bon équilibre sur une sorte de balancier entre l'absence et l'excès de formalisation comptable. Mais pour trouver le bon équilibre, choisir l'outil comptable adéquat, le modeler, le bricoler dirait Weick (1995), ou savoir l'abandonner quand il ne permet pas de faire face à la situation, un certain degré d'expertise, que le dirigeant de PME ne possède pas toujours, est requis. Les professionnels comptables internes et externes peuvent contribuer à l'émergence de cet équilibre en aidant le dirigeant à sélectionner, adapter et analyser les informations et les connaissances comptables utiles pour l'action.

La prudence doit cependant présider à l'interprétation et à l'appréciation de nos conclusions compte tenu des limites de l'étude. Ces limites sont de plusieurs ordres. Elles sont tout d'abord liées à la taille de l'échantillon : la constitution d'un échantillon de 92 observations directes en face à face sur un thème considéré sensible par les répondants, constitue un travail important pour le chercheur mais le statisticien le trouvera trop limité. Cet échantillon est par ailleurs concentré dans une seule ville (Alep). Ensuite, pour des raisons de praticabilité, certains facteurs de contingence susceptibles d'influencer la complexité du SIC des dirigeants de PME n'ont pu être pris en compte ; ainsi les aspects psychologiques de la personnalité du dirigeant ou encore l'influence de la culture ou de l'histoire de l'entreprise, n'ont pas été intégrés au modèle.

$\mathrm{Au}$ total, ces limites indiquent quelques directions de prolongements pour notre recherche. A notre sens, un approfondissement de la connaissance du comportement des dirigeants de PME face aux données comptables de gestion passe tout d'abord par un élargissement du modèle de recherche pour affiner et compléter certaines de nos conclusions. Le dirigeant de PME pourra notamment être caractérisé par des variables plus complexes et plus subjectives que celles utilisées dans notre étude. Les aspects psychologiques de sa personnalité (son attitude face au risque, sa culture, sa créativité) pourront être intégrés au modèle. Il passe ensuite par une multiplication d'analyses plus en profondeur dans les PME. Une étude qualitative permettrait notamment de mieux comprendre comment, avec quelques données comptables que l'on pourrait qualifier d'élémentaires dans le sens où elles constituent la base de ce qui est essentiel, une fraction significative de dirigeants de PME parvient à des représentations riches et variées.

\section{Bibliographie}

ABDEL KADER, M., R. LUTHER (2008), « The impact of firm characteristics on management accounting practices », British Accounting Review, vol.40, n 1 , p. 2-27.

AITA, S. (2007), «L'économie de la Syrie peut-elle devenir sociale ? Vous avez dit «Economie sociale de marché »? », In La Syrie au présent : Reflets d'une société (Dupret, B., Z. Ghazzal, Y. Courbage et M. Al-Dbiyat), France, Edition Sindbad/Actes Sud, chapitre 5, p. 543-624.

AFFÈS, H. et A. CHABCHOUB (2007), « Le système d'information comptable : les déterminants de ses caractéristiques et son impact sur la performance financière des PME en Tunisie », La Revue des Sciences de Gestion, Direction et Gestion, ${ }^{\circ} 224-225$, p. 59-68.

AL-OMIRI, M. et C. DRURY (2007), «A survey of factors influencing the choice of product costing systems in UK organizations », Management Accounting Research, vol.18, $\mathrm{n}^{\circ} 4$, p.399-424.

BARLETTE Y. et A. JAOUEN (2012), «Quelle est l'influence de l'expert-comptable sur le dirigeant de TPE ? Le cas de la gestion des informations de l'entreprise », $11^{\text {ème }}$ CIFEPME, Brest.

BELDI A. et W. CHEFFI (2005), « La comptabilité de gestion : outil d'information ou dispositif de connaissance ? La perception des managers », $26^{\text {ème }}$ Congrès de l'AFC, Lille, mai. 
BERGERON, H. (1996), Différenciation des systèmes de données et représentations en contrôle de gestion : essai d'observation et d'interprétation, Thèse de doctorat, Université de Montpellier II.

BESCOS P.L. et C. MENDOZA (1998), «Les besoins d'information des managers sont-ils satisfaits », Revue Française de Gestion, novembre-décembre, p.117-128.

BESCOS, P. L., E. CAUVIN, P. LANGEVIN et C. MENDOZA (2004), « Critiques du budget : une approche contingente », Comptabilité Contrôle Audit, vol. 10, n 1, p. 165-185.

BOULIANNE, E. (2007), «Revisiting fit between AIS design and performance with the analyzer strategic-type », International Journal of Accounting Information Systems, vol. 8, p. 1-16.

BRUNS, W.J. et J.H. WATERHOUSE (1975). «Budgetary control and organization structure », Journal of Accounting Research, Autumn, p.177-203.

CHAPELLIER, P. (1996), «Données comptables de gestion et système d'information du dirigeant de PME », Système d'Information et Management 2 (1): 23-41.

CHAPELLIER, P. (2003), «Les apports d'Internet à la mission de l'expert-comptable dans les petites entreprises », Comptabilité-Contrôle-Audit, vol. 9, n 2, p. 171-187.

CHEFFI, W. et M. NEKHILI (2011). «Rôles assignés à la comptabilité de gestion par les managers et changement comptable : question d'adéquation ou simple désillusion ? », Comptabilité, Contrôle, Audit, tome 17, Vol.1, p.67-97.

CHENHALL, R.H. (2003), «Management control systems design within its organizational context: findings from contingency-based research and directions for the future », Accounting, Organizations and Society, vol. 28, $\mathrm{n}^{\circ} 2-3, \mathrm{p} .127-168$.

CHENHALL, R.H. et K. LANGFIELD-SMITH (1998). «The relationship between strategic priorities, management techniques and management accounting: an empirical investigation using a systems approach », Accounting, Organizations and Society, Vol. 23, No 3 : 243-264.

COKER, J.W. et R.D. HAYES (1992). "Lenders' Perceptions of Income-Tax-Basis Financial Reporting by Small Business », Journal of Small Business Management, Vol. 30, № 3 : 66-76.

COLOT, V. et P.A. MICHEL (1996), «Vers une théorie financière adaptée aux PME : Réflexion sur une science en genèse », Revue Internationale PME, vol. 9, n 1, p. 143-166.

COUTERET P., et J. AUDET (2006), «Le coaching, comme mode d'accompagnement de l'entrepreneur», Revue Internationale de Psychosociologie et de Gestion des Comportements Organisationnels, 27, Vol XII, p.139-157.

COUTERET P. (2010), «Peut-on aider les entrepreneurs contraints ? Une étude exploratoire », Revue de l'Entrepreneuriat, Vol 9.2, p.6-33.

CROZIER M. et E. FRIEDBERG (1977), L'acteur et le système, Paris, Edition du Seuil.

DAVILA, T. (2005), « An exploratory study on the emergence of management control systems: formalizing human resources in small growing firms », Accounting, Organizations and Society, 30 (3): $223-248$.

EVRARD, Y., B. PRAS, et E. ROUX (2009), Market : fondements et méthodes de recherches en marketing, 4 ème édition, Paris, Edition Dunod.

FLACKE, K. et K. SEGBERS (2005), « Does managerial accounting follow entrepreneurial characteristics? Results of an empirical analysis of german SME », working paper, Univ of Munster GERMAIN C. (2001), «Le pilotage de la performance dans les PME, les résultats d'une enquête empirique », $22^{\text {ème }}$ congrès de l'Association Francophone de Comptabilité, Metz, Mai.

GERMAIN C. (2004), «La contingence des systèmes de mesure de la performance : les résultats d'une recherche empirique sur le secteur des PME », Finance, Contrôle, Stratégie, 7 (1) : 33-52.

HALDMA, T., K. LÄ̈̈TS (2002)« Contingencies influencing the management accounting practices of Estonian manufacturing companies », Management Accounting Research, vol.13, n4, p.379-400

JÄNKÄLÄ, S. (2007). Management control systems (MCS) in the small business context : Linking effects of contextual factors with MCS and financial performance of small firms, Oulu Univ Press.

JOLIBERT, A., et P. JOURDAN (2006), Marketing Research : Méthodes de recherche et d'études en marketing, Paris : Dunod.

JULIEN P.A. (1997), « Pour une définition des PME », in P.A. Julien (Éd.), Les PME : Bilan et perspectives, Economica.

KHANDWALLA, P.N. (1972), "The effects of different types of competition on the use managerial controls », Journal of Accounting Research, vol. 10, ${ }^{\circ}{ }^{2}$, p. 275-285.

LAVIGNE B., (2002), "Contribution à l'étude de la genèse des états financiers des PME », Comptabilité, Contrôle, Audit, Tome 8, Vol. 1, mai, pp. 25-44.

LAVIGNE, B. et J. SAINT-PIERRE (2002), «Association entre le système d'information comptable des PME et leur performance financière », Actes du $6^{\text {ème }}$ CIFEPME, Montréal. 
MALHOTRA, N. (2007), Etudes marketing avec SPSS, 5 ème édition, Harlow, Pearson Prentice Hall. MALO J.L. (1989), «Première approche d'un cadre méthodologique de recherche en comptabilité », 10 ème Congrès de l'Association Francophone de Comptabilité, Reims, p.384-420. MARCHESNAY, M. (1992), La PME : une gestion spécifique ? Problèmes économiques, vol. 2.276, p. 26-32.

MARCHESNAY M. (2003), «La petite entreprise : sortir de l'ignorance », Revue Française de Gestion, Vol 29, n²144, mai-juin, p.107-118.

MARRIOTT, N. et P. MARRIOTT (2000), «Professional accountants and the development of a management accounting service for the small firm : barriers and possibilities ", Management Accounting Research, vol. 11, $\mathrm{n}^{\circ}$ 4, p. 475-492.

MINTZBERG, H. (2006). Le manager au quotidien : les dix rôles du cadre, Ed. d'Organisation.

NANDAN, R. (2010), "Management Accounting Needs of SMEs and the Role of Professional Accountants: A Renewed Research Agends », Journal of Applied Management Accounting Research, vol. 8, $\mathrm{n}^{\circ} 1, \mathrm{p} .65-77$.

NAYAK, A. et S. GREENFIELD (1994), « The use of management accounting information for managing micro businesses », In Finance and the Small Firm, London, Routledge, p.182-231.

NGONGANG, D. (2007), « Analyse des facteurs déterminants du système d'information comptable et des pratiques comptables des PME tchadiennes », Revue des Sciences de Gestion, N²24-225.

NOBRE T. (1999), "Des méthodologies de recherche pour repenser le contrôle de gestion », in Dupuy Y., Faire de la recherche en contrôle de gestion ?, Vuibert, p.145-160.

NOBRE, T. (2001), «Méthodes et outils du contrôle de gestion dans les PME », Finance Contrôle Stratégie, vol. 4, n 2 , p. 119-148.

NYENGUE EDIMO, P. (2006), L'organisation du système d'information comptable des entreprises camerounaises : essai d'observation et interprétation des pratiques. Thèse de doctorat en sciences de gestion, Bordeaux, Université de Bordeaux IV.

RAYMOND, L., BLILI, S., et D. EL-ALAMI (2004), «L'écart entre le consultant et la P.M.E. : analyse et perspectives », Gestion, vol. 28, n 4 (hiver), p. 52-60.

ROWE, F., V. FERNANDEZ et C. PICORY (1994). "Diversité, cohérence et pertinence des outils

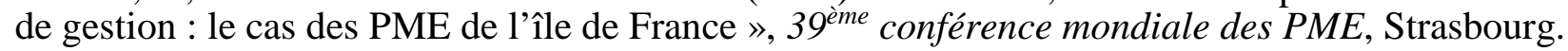

SABOLY, M. (1994), Les déterminants de la qualité des produits comptables : le rôle du dirigeant, Thèse de doctorat en sciences de gestion, Poitiers, Université de Poitiers.

SAINT-PIERRE, J. et M. BAHRI (2003). «Relations entre la prime de risque bancaire des PME et les composantes de leur risque global », Association Canadienne des Sciences Administratives, Halifax, Juin.

SANDRINO, T. (2007), «Introduction the First Management Control Systems : Evidence from the Retail Sector », The Accounting Review, Vol. 82, N $1: 265-293$.

SANTIN, S. et D. VAN CAILLIE (2008), «Le design du système de contrôle de gestion des PME: une quête de stabilité adaptative », $29^{\text {ème }}$ Congrès annuel de l'AFC, Paris, Mai.

SATHE, V. (1975) «Contingency theories of organizational », In Managerial Accounting: The Behavioural Foundations (Ed, Livingstone, J. L.). Colombus: Grid, 51-63.

TELLER, R. (1999). Le contrôle de gestion: Pour un pilotage intégrant stratégie et finance. Caen : Editions Management et Société.

THIETART, R.A., et coll. (2007), Méthodes de recherche en management, $3^{\text {ème }}$ éd., Ed. Dunod.

TILLEMA, S. (2005), « Towards an integrated contingency framework for MAS sophistication: Case studies on the scope of accounting instruments in Dutch power and gas companies », Management Accounting Research 16 (1): 101-129.

TORRES O. (2004), Essai de théorisation de la gestion des PME: de la mondialisation à la proxémie, Habilitation à Diriger des Recherches, soutenue à l'Université de Caen.

TOUCHAIS, L. (1998), « Le contrôle de gestion et l'exportation : une combinaison originale de pratiques formelles et informelles », Finance Contrôle Stratégie, Vol. 1, № $4: 151-171$.

VALLERAND J., S. BERTHELOT, et J. MORRILL (2008), «Positionnement de la PME manufacturière canadienne face aux outils de gestion enseignés dans les programmes de formation universitaire en administration », $9^{\text {ème }}$ Congrès International Francophone PME, Louvain la Neuve. WEICK K.E. (1995), Sensemaking in organizations, Sage.

ZAWADZKI C. (2011) «L'évolution des routines organisationnelles en PME : leçons de l'échec d'une démarche d'introduction du contrôle de gestion »,32e Congrès de l'AFC, Montpellier, Mai. 